\title{
La conferencia en la escuela primaria
}

\author{
Itziar Plazaola
}
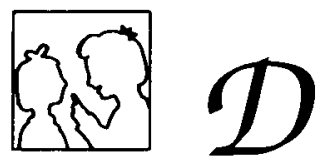

Se analiza cómo una actividad muy frecuente en la escuela primaria como es la conferencia plantea serios problemas a los alumnos, ya que no están previstas secuencias didácticas para su enseñanza. A parte de lo imprecisas que resultan las relaciones entre las actividades escritas (tomar notas, preparar un plan, etc.) y la actividad oral propia de la conferencia (exponer oralmente un tema), los alumnos se enfrentan a textos enciclopédicos que les resultan, la mayoría de las veces, incomprensibles.

\section{PRESENTACION}

En la escuela primaria de la Suiza francófona existe una tradición de realización de conferencias. Los alumnos, en la mayoría de los casos a partir del cuarto año, preparan una conferencia que presentarán oralmente, llegado su momento, ante la clase.

Esta práctica escolar no obedece a ninguna propuesta concreta hecha por los manuales o por los programas oficiales. Ni en los programas anteriores a la renovación de la enseñanza de la lengua que tuvo lugar en los años 70 , ni en los actuales, existe referencia alguna a esta práctica. Se trata de una actividad que tiene su origen en la pedagogía de inspiración freinetiana como el texto libre o el diario escolar redactado por los alumnos. El/la maestro/a recurre a la conferencia por convicción personal o bien para variar el tipo de actividades realizadas en clase. De esta manera, la conferencia como ocurre con ciertas «costumbres escolares», perdura a través de renovaciones y programas.

Actualmente, en la reflexión sobre la didáctica de la lengua oral, la conferencia aparece de manera marginal. Los autores la citan como un caso de actividad oral, pero de una lengua oral muy próxima a la escrita por lo que aparece con cierto matiz negativo, como si se tratara de una «falsa» lengua oral. Esto ocurre por exemplo con un autor como Perrenoud (1991), que plantea la falta de referencias didácticas existentes en la enseñanza de la lengua oral. Ante esta situación, el autor advierte del riesgo que existe en trasladar hacia la lengua oral las normas de corrección existentes para la lengua escrita, lo que favorecería necesariamente una lengua oral poco interactiva, y da como exemplo la conferencia. 
En nuestra opinión, nada justifica que la conferencia desaparezca de la escuela y esta tarea escolar «tradicional» puede, a la luz de las corrientes discursivas actuales, tener una nueva vida a condición de que sea tratada con la atención que requiere.

\section{¿QUE SE ENTIENDE POR CONFERENCIA?}

La primera pregunta que se nos plantea es qué se entiende por conferencia, y más concretamente qué entiende, por un lado, el/la maestro/a y, por otro lado, qué entienden los alumnos.

Según nuestros datos cuando el maestro/a propone esta actividad no acostumbra explicar, más que de una manera un tanto vaga, cuáles son las características del tipo de discurso del que se trata, ni se suele efectuar ningún tipo de ejercicio previo, como lectura, reconocimiento y observación de textos informativos, explicativos, etc.

Podemos suponer que el maestro/a va a exigir que los alumnos produzcan un texto del que conoce un «arquetipo" gracias a las conferencias a las que ha podido asistir. Más difícil resulta tratar de reconstruir cual puede ser la representación que poseen los alumnos sobre este discurso, habida cuenta de que la gran mayoría de los alumnos de estas edades no ha tenido ocasión de asistir jamás a una conferencia.

Si nos atenemos al análisis que realiza Goffman de la conferencia (Goffman, 1987), ésta se define en términos globales como una ocupación oficial y prolongada de una escena. El estilo del conferenciante es de ordinario serio y un tanto impersonal. En la mayoría de los casos existe un estrado, lo que pone de manifiesto el hecho de que el público forma un auditorio inmediato. De las personas que se presentan ante dicho auditorio se dice que realizan una representación en el sentido teatral de la expresión. Lo que hace suponer que dichas personas poseen capacidades escénicas cuya ausencia haría enredarse lamentablemente al individuo ordinario proyectado sobre una escena. En esto se opone a la conversación cotidiana en la que, se supone, nadie pretende un papel preponderante. Prosiguiendo con su análisis, Goffman pone de manifiesto la importancia de los aspectos ligados al «acontecimiento», al lado de los aspectos ligados a la conferencia propiamente dicha.

En cuanto al interlocutor, en el caso de la conferencia se considera que es la misma persona que el autor (que ha redactado y escrito el texto), y que además cree personalmente en lo que dice, es decir que adopta el punto de vista expuesto.

Por otra parte, se atribuye a este triple agente una "autoridad intelectual», por oposición a institucional. Se le supone poseedor de conocimientos y experiencia en la materia de un modo muy superior al de su público.

Ateniéndose a algunas de las características textuales de la conferencia, Goffman señala la importancia de lo que él llama el «marco» del texto. Se trata por una parte de la introducción y conclusión, y por otra parte de los «comentarios parentéticos», es decir de los ejemplos, disgresiones, extensiones etc., que aparecen a lo largo de la conferencia. Estos elementos de encuadre tienen un gran interés del punto de vista de la interacción, puesto que están orientados hacia el texto, y al mismo tiempo obedecen a los intereses e identidad de un público particular. Se trata de modos de ajuste utilizados por el interlocutor para adaptar su texto al público concreto a medida que transcurre la conferencia y gracias a su percepción de las reacciones del auditorio.

Este modo de análisis de un hecho discursivo nos parece que posee el mérito de permitir poner de relieve algunas de las reglas del juego, y la conferencia aparece así perfilada en lo que se refiere a sus caractéristicas socio-discursivas, y también a algunas de las particularidades textuales o lingüísticas. 


\section{ENTRE LO ESCRITO Y LO ORAL; ¿QUÉ LUGAR O DONDE SITUAR LA CONFERENCIA?}

Antes de pasar a observar cuales son los paralelismos, si es que existen, entre la conferencia como género discursivo y la práctica escolar, intentaremos aportar algunas precisiones sobre el estatuto de la conferencia.

En la presentación, hemos hablado de la idea de algunos autores que consideran la conferencia como una producción textual en la que priman las normas del escrito, «solo que» se realiza, se produce, oralmente. Dicha concepción nos parece poco satisfactoria y el análisis de Goffman ha permitido precisar las características de este discurso.

Este autor habla también de la existencia en nuestra sociedad de tres maneras de animar las palabras pronunciadas: la memorización, la lectura en voz alta y el habla espontánea. De estas tres formas de producción, la memorización se emplea en ciertos casos en las conferencias, pero sin reconocerlo. La lectura en voz alta es una modalidad frecuente y el habla espontánea aparece como un ideal, con frecuencia realizado con ayuda de notas. De todos modos, la modalidad de habla espontánea contiene una buena dosis de ilusión. Esta modalidad es sin duda la más adecuada para el marco de la conferencia (introducción, conclusión y comentarios parentéticos) y tiene la ventaja de producir un efecto de adaptación del texto a la situación presente, haciéndolo así aparecer como original. Un método clásico que permite la simulación del habla espontánea es el de leer un párrafo que se reproduce, acto seguido, en voz alta como si se tratara de una improvisación.

En la emisión de un conferencia se busca un efecto de flujo verbal regular evitando cuidadosamente los titubeos, vueltas atrás, errores de dicción, etc., que sin embargo caracterizan el habla espontánea que se trata de simular, lo que constituye una prueba del carácter específico del habla del conferenciante.

\section{ANALISIS DE LAS DIVERSAS TAREAS QUE SUPONE LA PREPARACION Y REALIZACION DE UNA CONFERENCIA}

A continuación nos centraremos en la práctica escolar de la conferencia, para analizar las principales fases de dicha acrividad. En cada una de ellas presentamos algunos resultados de las investigaciones y reflexiones que las corrientes de la didáctica del texto han realizado en los últimos años.

\section{LA LECTURA DE LOS DOCUMENTOS}

Una vez definido el tema del que va a tratar, el alumno recurre a uno o varios documentos para la búsqueda de información. En general estos documentos le son facilitados por el maestro mismo o se obtienen en la biblioteca escolar.

La temática más común en este tipo de actividades es la del mundo animal: el león, la salamandra, el hámster, etc. son los temas recurrentes. Para documentarse sobre estos temas existen, además de las publicaciones especializadas, un cierto número de enciclopedias destinadas a un público joven.

Podemos considerar que la enciclopedia para jóvenes a la que el alumno recurre, pertenece al tipo informativo. De hecho, las diferencias entre las distintas obras son considerables. Algunas veces se trata de obras de vulgarización próximas a las realizadas para un público adulto. En otros casos, el texto tiende más al tipo explicativo, con una parte de problematización que conlleva preguntas que el autor tratará 
de responder a lo largo de su artículo. Sin entrar en mayores detalles sobre las diferencias entre las diversas enciclopedias al alcance del alumno, centrémonos en el caso general del texto informativo.

Autores como Combettes y Tomassone (1988) analizan algunas de las particularidades del texto informativo con el fin de comprender mejor las dificultades que encuentran los alumnos en la lectura de estos textos.

Se cree con frecuencia que la dificultad de lectura de los textos informativos está ligada al léxico y a la complejidad sintáctica de sus enunciados. A este presupuesto, los autores contraponen la constatación de que las dificultades surgen también con estructuras de frase sencillas, y concluyen que al menos una parte de los problemas tiene su origen en los aspectos propiamente informativos del enunciado. Dos son las nociones que interesan aquí: la noción de «conocimiento compartido", es decir la parte de la información que el autor considera que conoce su destinatario y la distribución de la información o «densidad de información» de los diversos enunciados. Según estos autores la estructuración sintáctica y textual de un texto informativo depende de estos dos factores. En ellos reside también una gran parte de las dificultades de interpretación.

Este breve resumen pretende mostrar que cuando el análisis se centra en las características del texto informativo, nos vemos obligados a abandonar el optimismo consistente en creer que para que los alumnos puedan completar sus conocimientos sobre un tema, basta con proporcionarles ciertas publicaciones de tipo informativo.

\section{La toma de notas}

El alumno deberá a continuación tomar notas de la(s) enciclopedia(s) utiliza$\mathrm{da}(\mathrm{s})$.

En los últimos años son varias las investigaciones que se han ocupado de la toma de notas. Los datos obtenidos son de un gran interés para nuestra reflexión. Garcia-Debanc (1985) presenta una investigación realizada con alumnos de 9 años en la que trata de identificar las dificultades encontradas por los alumnos y propone ejercicios destinados a mejorar los resultados, en una óptica de evaluación formativa. Los principales problemas surgidos ante la toma de notas son:

1. La aparición de dificultades en la comprensión del documento utilizado.

2. La dificultad de selección de las informaciones pertinentes. La lentitud en la escritura constituye un handicap que debe ser compensado por una selección pertinente de las informaciones.

Pero dicha selección se ve entravada por:

- el olvido de la consigna; puede ocurrir que el alumno cambie de tema en su búsqueda orientado por sus propios centros de interés.

- con frecuencia los alumnos copian fragmentos enteros del documento, incluyendo palabras que no entienden.

3. Las dificultades ligadas a la jerarquización de las informaciones. Para poder organizar jerárquicamente las informaciones en la toma de notas, es necesario agrupar en un solo párrafo las diversas informaciones que se refieren a un mismo aspecto. Esto resulta difícil para muchos alumnos. La elección de un término o expresión genérica como título de las informaciones recogidas constituye una nueva dificultad. Con frecuencia los alumnos muestran que han buscado un título pero los medios lingüísticos de los que disponen les inducen al error y proponen nominalizaciones inexistentes o no aplicables al tema.

\section{La redacción de la conferencia}

En algunas de las clases en las que se practica la conferencia, los alumnos se presentan con notas tomadas en sus lecturas y realizan oralmente su intervención. 
En dicha situación, el alumno deberá recrear el entorno textual del dato que ha anotado, adaptándolo a su texto. A medida que produce los enunciados debe controlar la coherencia global, con las consiguientes cadenas anafóricas, respeto de los tiempos verbales, conexión, etc. que requiere su texto. Este tipo de actividad ha sido poco estudiado dadas las dificultades que plantean las investigaciones sobre la producción oral.

En otros casos, los alumnos pasan por una fase de redacción del texto antes de presentarlo ante la clase.

La bibliografía existente sobre los procesos que intervienen en la redacción es numerosa y da cuenta de la amplitud del tema. Pretender enumerar las diversas operaciones psicolingüísticas que el sujeto realiza a lo largo de una actividad redaccional es, hoy por hoy, presuntuoso. Haciendo abstracción de las diferentes corrientes, terminologías y clasificaciones a los que los autores se refieren en este tema, resumimos en dos grandes grupos las principales operaciones (no tratamos aquí de las operaciones de contextualización que tratan de los aspectos sociales y enunciativos de la producción):

- Las operaciones de planificación que agrupan la activación, selección y composición de conocimientos de todo tipo que dan lugar a un plan de texto, el cual dirige la actividad redaccional en su conjunto.

- Las operaciones que intervienen en la textualización que tiene lugar a nivel local y en las que intervienen la gestion de los indicadores temporales, de determinación, tematización, conexión, etc.

Los psicolingüistas hablan de problemas de «sobrecarga cognitiva» para referirse a la dificultad que tiene su origen en la acumulación de las muy numerosas tareas que comprenden ciertas actividades.

En respuesta a esta nueva dificultad, los especialistas tratan de imaginar técnicas destinadas a ayudar al sujeto. Los autores proponen por ejemplo entrenar al sujeto en una de las operaciones que intervienen en la tarea, en lugar de someterlo constantemente al tratamiento del conjunto de las operaciones. Se considera que la lentitud y la dificultad en la realización de ciertas operaciones locales puede entorpecer gravemente el conjunto de la actividad. Para la didáctica del texto es fundamental el obtener el aislamiento de las operaciones que permita crear ejercicios destinados a mejorar las diferentes capacidades implicadas. Existen algunas propuestas orientadas hacia el desarrollo de las capacidades de reformulación, de definición de las orientaciones argumentativas, etc.

\section{PRIMERAS CONCLUSIONES}

El estudio sociolingüístico de Goffman y las referencias citadas de la psicolingüística y de la didáctica del discurso permiten dar un nuevo enfoque a la cuestión de la conferencia en la escuela. Podemos pues concluir que:

a. Las características socio-discursivas de la conferencia, con su funcionamiento ritualizado, su carácter de exhibición de conocimientos y habilidades discursivas del conferenciante, no parecen corresponder a la actividad que se plantea habitualmente en las escuelas.

b. En el análisis que hemos efectuado de la actividad escolar (que por comodidad seguiremos, de momento, llamando conferencia), esta aparece compuesta de tres fases: la lectura de los documentos, la toma de notas y la producción oral o escrita. Cada una de estas tareas ha sido estudiada por autores que han puesto de relieve la complejidad que conllevan. Complejidad que se traduce, primeramente en el elevado número de 
operaciones que intervienen en su realización, y también en las dificultades que la realización de estas tareas plantean a los alumnos incluso de niveles de escolarización muy superiores a los de la primaria.

c. El descubrimiento de la diversidad de las operaciones implicadas en las distintas fases de elaboración, permite imaginar subtareas, situaciones de producción, etc. que favorezcan el ejercicio «por separado» de las capacidades en juego.

\section{LA CONFERENCIA A PARTIR DE NOTAS: UN EJEMPLO}

Presentamos a continuación una secuencia didáctica sobre la conferencia, tal y como se realiza en una escuela ginebrina con alumnos de $5^{\circ}$ y $6^{\circ}$ año .

Esta secuencia cubre dos cursos y se presenta en cinco fases.

$\begin{array}{ll}\text { Fase } & \text { Duración } \\ 1 & 30 \mathrm{sg}-1 \mathrm{~min} \\ 2 & 1 \mathrm{~min}-3 \mathrm{~min} \\ 3 & 5 \mathrm{~min} \\ 4 & 10 \mathrm{~min} \\ & \\ 5 & 10 \mathrm{a} 20 \mathrm{~min}\end{array}$

Notas

Algunas palabras

Algunas palabras

Plan con algunas palabras o cifras

Plan con algunas notaso cifras

Resumen para los compañeros

Plan con algunas notas o cifras

Resumen para los compañeros
Documento

Ninguno

3 documentos

Máx. 5 documentos

Máx. 10 documentos

Documentos foto-

gráficos y documentos

Las dos primeras fases tienen lugar durante el $5^{\circ}$ año y el resto al año siguiente. Para las tres primeras fases el tema propuesto es «un animal», en las últimas fases el tema es libre. En las primeras tres fases, la conferencia es oral, con ayuda de unas notas. En cambio, en los dos últimos casos el alumno debe además preparar un documento que contenga una foto, dibujo o esquema y un resumen de media página. Este resumen está destinado a los otros alumnos de la clase, pero el alumno no lo tiene presente durante la conferencia.

Es frecuente que el maestro/a proponga un plan de trabajo o una serie de consejos que pueden incluir un plan de texto. Los consejos están relacionados con la necesidad de preparar el trabajo con antelación, de buscar el significado de todos los términos que van a emplear. etc.

De este ejemplo podemos retener algunos aspectos positivos. Frente a otros casos en los que la conferencia constituye una actividad aislada y a veces única durante el año escolar, este caso propone cierta progresión y continuación durante dos años. Por otra parte, el maestro propone como ayuda un plan del que el alumno se sirve en la búsqueda y selección de la información, en la estructuración de su producción y en la conferencia propiamente dicha, es decir, durante la toma de palabra ante la clase.

\section{UNA CONFERENCIA CON TEXTO ESCRITO ${ }^{2}$}

Para poder llevar a cabo una observación más detallada de los resultados obtenidos por los alumnos en la realización de una conferencia, hemos recurrido a otro centro escolar. En una clase de $5^{\circ}$ curso, los alumos en grupos de 3 ó 4 preparan una conferencia sobre un animal que ellos han elegido. Para ello deben realizar un texto escrito siguiendo un plan facilitado por la maestra. La existencia de una fase escrita en la realización de esta actividad hace posible el reunir y comparar los trabajos escritos por los alumnos y los documentos utilizados para su preparación.

El análisis de estos textos y documentos nos ha permitido aislar algunos de los problemas a los que los alumnos han tenido que enfrentarse en la realización de la tarea. 
La cuestión de las enciclopedias

Las obras utilizadas para reunir la información plantean una serie de dificultades. En muchos casos, la publicación que los alumnos tienen a su disposición trata el tema de una manera muy extensa y con un nivel científico muy superior al nivel de los conocimientos de los niños. Son varios los aspectos a señalar:

a) Los títulos

En la mayoría de los ejemplos, los capítulos del plan de los alumnos no aparecen como títulos del artículo de la enciclopedia. Esto supone que incluso con un plan, la búsqueda de la información correspondiente a un capítulo resulta complicada. Por otra parte, los títulos de la enciclopedia no siempre son comprensibles para el alumno, y en consecuencia la decisión de si puede o no resultar informativo para su capítulo, es difícil de tomar. Veamos un ejemplo de un trabajo sobre el tiburón.

\section{Descripción física}

En los tiburones las branquias se hallan contenidas en cinco cámaras abiertas por un lado hacia la faringe, y por el otro sobre los costados de la cabeza del animal.

Un solo detalle ha cambiado radicalmente: la boca se ba desplazado tomando una posición ventral, bastante incómoda para comer ${ }^{3}$

La primera parte de este párrafo ha sido tomada palabra por palabra de un capítulo titulado, «Cómo respiran los tiburones». Las tres líneas finales pertenecen a un capítulo titulado, "La rotación de la boca y el olfato en los tiburones» que hace referencia a los aspectos de la morfología del animal que han evolucionado. Aquí, el alumno ha retenido que el capítulo trataba de la boca y el olfato, es decir de sus características físicas, pero la idea de que algunos de los aspectos morfológicos hayan podido evolucionar sobrepasa sus conocimientos. Por otra parte, las dificultades de comprensión del texto no le permiten caer en la cuenta del error cometido.

b) Las variedades dentro de la misma especie

Otra de las dificultades relacionadas con las enciclopedias, sobre todo cuando se trata de flora o de fauna, viene del hecho de que la información sobre una especie determinada aparece en alternancia constante con informaciones sobre las variedades existentes al interior de la misma especie, variedades cuyas denominaciones no coinciden siempre exactamente, de una publicación a otra.

Así en un trabajo sobre la serpiente cobra, una alumna propone:

\section{Habitat}

Cobra egipcia: vive en las regiones secas del Africa septentrional y oriental, así como en el suroeste de Asia.

Cobra de cuello negro: vive en las sabanas.

Cobra blanqui-negra: más pacífica y vive en los bosques.

Cobra india: vive en toda Asia meridional desde Irán hasta el sur de China y en Indonesia. La cobra india construye un nido.

Cobra real: vive en los desiertos egipcios y en Arabia. Vive también en China, sur de Vietnam, Birmania, Malasia y en Filipinas.

Cobra acuática de Africa tropical: vive en África tropical.

La serpiente de Cleopatra: vive en Arabia y en el noroeste de África.

Cobra con monóculo: vive en la península malasia y en los confines orientales del Asia central.

La serpiente con gafas: vive en las regiones de Asia central.

Cobra de Asia central: vive incluso hasta en la India occidental.

Cobra egipcia: vive en casi todas las regiones semi-desérticas de África. 
P.D.: Algunas cobras ban abandonado la superficie del suelo y viven en el agua y en los àrboles.

La selección de las informaciones principales plantea serias dificultades, y el alumno se pierde (ver repetición) ante tal diversidad dando lugar a un texto que retiene unos conocimientos de dudoso interés para estas edades.

El recurso a un listado de informaciones recopiladas en diversos libros de referencia constituye una ilustración de la ausencia total de resumen y reformulación del que sufren estos trabajos. Este ejemplo muestra, por otro lado, la dificultad que presenta la selección de las informaciones principales frente a las secundarias. De hecho en un tema como el habitat de una especie animal (así como cuando se trata de las dimensiones, color, etc.), es frecuente que las enciclopedias muestren la diversidad existente al interior de las diversas variedades animales lo que constituye un nuevo obstáculo.

c) Los «trucos»

En otros ejemplos se puede observar que el alumno/a elije el texto que acompaña una ilustración. Este hecho nos parece significativo de la dificultad que presenta la lectura y el tratamiento del artículo, al que el alumno prefiere el texto conciso de la ilustración. Pero una práctica indiscrimanada de esta técnica, junto con el problema de comprensión que aparece como una constante, pueden dar lugar a algunos ejemplos curiosos. En un texto sobre la salamandra aparece una utilización frecuente del «truco» del texto de la ilustración, y el capítulo del alumno termina así:

La salamandra puede tener una coloración variable de un individuo a otro. Posee una vista dorsal y ventral.

El texto de la enciclopedia decía así:

La salamandra de manchas tiene una coloración variable de un individuo a otro. Serie de variaciones, vista dorsal y vista ventral de los mismos animales.

Texto que acompaña a dos ilustraciones que representan varias salamandras fotografiadas en posición dorsal en la primera fotografía y ventral en la segunda.

En resumen, la característica principal de los textos que producen los alumnos en esta situación es la de un «patchwork» compuesto por frases tomadas literalmente de los textos de referencia sin apenas transformación excepto, algunos casos de adaptación del sujeto del enunciado. No existe pues, lo que se ha dado en llamar reenunciación, en la que un segundo enunciador, en nuestro caso, el alumno/a produce enunciados a partir de las informaciones obtenidas en el texto de referencia, no hay parafrase, no hay reformulación.

\section{A MODO DE CONCLUSION}

Trataremos a continuación de resumir algunas de las conclusiones a las que nos han conducido las reflexiones y observaciones realizadas aquí. Frente a las notables diferencias que han aparecido entre la práctica social de la conferencia y la actividad escolar del mismo nombre, tres son las opciones que se dibujan:

1. La primera opción consiste en elejir como actividad escolar una situación de producción de texto que voluntariamente haga eco a la conferencia. En este caso, parece imprescindible realizar un trabajo preparativo en clase destinado a mostrar las particularidades de dicho género discursivo. Algunas de las características señaladas por Goffman nos parecen especialmente importantes. Pensamos en las que se refieren a la posición del interlocutor respecto a su texto, a saber la adopción por parte del interlocutor del punto de vista de su texto, haciéndolo suyo. Para que este «hacer 
suyo» el texto sea posible nos parece que habría que elegir temas muy próximos a la realidad del niño de manera que se pudiera sentir, frente al público de sus compañeros, como un especialista en la materia. Para ello tiene que preparar un texto de tipo informativo, en este sentido el alumno deberá enriquecer sus conocimientos sobre el tema elegido recurriendo a una bibliografía adecuada. Además, habrá que desarrollar en clase un trabajo sobre el «marco de la conferencia» (los comentarios destinados a adaptar el texto al público y al aquí y ahora) que requiere una presentación y reflexión.

Habría que tratar en clase también la cuestión de la "falsa» habla espontánea, proponiendo a los alumnos la preparación de un texto (escrito de prosa oral), al que se le añaden de manera más o menos espontánea los elementos del llamado marco.

2. La segunda opción consiste en trabajar una forma de discurso de tipo «informe», es decir un texto informativo de carácter más o menos neutro y que no posea las particularidades de la conferencia. Es la opción que parece practicarse, sobre todo cuando se pasa por una fase escrita del texto.

Debemos señalar que cuando el maestro/a facilita un plan, la actividad resulta más sencilla ya que la tarea principal reside en el «rellenado» de los diferentes títulos propuestos. Esta facilitación parece absolutamente necesaria en particular en los niveles que nos ocupan.

En cambio, la propuesta de un tema libre nos parece que plantea problemas importantes sin que por otro lado posea grandes ventajas; nos referimos al problema de la documentación.

En efecto, aparece excesivamente arriesgado el dejar a los alumnos solos en la búsqueda de documentación. Los problemas ligados a las enciclopedias nos parecen centrales. En realidad, habría que partir de documentos controlados por el profesor, y sobre algunos de los cuales se efectúa un trabajo de lectura en clase. Para una realización más cómoda los manuales podrían proponer textos informativos sobre distintos temas (textos que pueden perfectamente no ser «auténticos» sino preparados, con un nivel de dificultad informativa controlado). Claro que no se podrá ofrecer una gran diversidad temática, pero esto nos parece aquí secundario.

La lectura y discusión en común de algunos textos, con el fin de identificar los diferentes aspectos del contenido (alimentación del animal, reproducción, etc.), nos parece un requisito previo a la fase de realización.

3. La tercera opción consistiría en adoptar una parte de las tareas que componen la preparación de la conferencia en objetivo en sí, por ejemplo: la toma de notas. Es cierto que esta tarea parece más adecuada a las capacidades del alumno/a a estas edades, es más, en las realizaciones de conferencias que hemos observado, de hecho los alumnos «se limitan» a una toma de notas gracias a su plan. Se puede afirmar que no hay producción de texto. En este sentido parece razonable proponer un tratamiento más simple, es decir, el de una parte de las operaciones implicadas en la tarea de preparación de una conferencia.

\section{Notas}

1 Nuestro agradecimiento a $M$. Gaugaz de la escuela de Onex-Parc.

2 Agradecemos a Mme Paineau del «Service du français» de Ginebra el habernos facilitado los documentos.

3 Para comodidad del lector, traducimos los textos originales escritos en francés. 


\section{Referencias}

Combettes, B., y Tomassone, R. (1988) Le texte informatif, aspectos linguistics, Bruselas, De Boeck-Wesmeel.

ChaROLES, M. (1978) «Introduction aux problèmes de la cohérence des textes», en Langue Française, 38, pp 7-41

CHAROLIES, M. (1986) «L'analyse des processus rédactionnels: aspects linguistiques, psycholinguistiques et didactiques", en Pratiques, 49

FAYOL, M. (1984) «Pour une didactique de la rédaction: faire progresser le savoir psychologique et la pratique pédagogique. L'approche cognitive de la redaction», en Repères, 63, pp. 65-69

GofFMAN, E. (1987) Fafons de parler, París, Minuit

GREGG, L.-W. y STEINBERG, E.-R. (Ed.) (1980) Cognitive process in uriting, Hillsdale, New Jersey, Lawrence Erlbaum Associates, Publishers

MASSERON, C. «La correction de rédaction», Pratiques, 29, pp. 47-68

Nique, C. y LeLIÈVRE, C. (1978) «Le texte écrit d'élève: production d'un sujet, ou produit de déterminations?", en Langue Française, 38, pp. 42-57

Petitjean, A. (1980) «L'atelier d'écriture», en Pratiques, 26

Petitjean, A. (1985) «Apprentissage de l'écriture et travail en projet», en Etudes de linguistique appliquée, 59, pp. 88-102

Perrenoud, Ph. (1991) «Bouche cousue ou langue bien pendue? L'école entre deux pédagogies de l'oral», en Wirthner, M.; Martin, D. y Perrenoud, Ph. (Ed.) Parole étouffée, parole libérée. Fondements et limites d'une pédagogie de l'oral, Neuchâtel-París, Delachaux et Niestlé, pp. 15-40

\section{La conferencia en la escuela primaria Itziar Plazaola CL\&E, 1994, 23, pp. 81-90}

Resumen: En la escuela francófona suiza una actividad frecuente es la conferencia. Se trata de una presentación oral preparada por cada alumno (o en pequeños grupos) sobre diversos temas. Esta tarea, aunque no se encuentra explícitamente en los programas, obedece a una tradición escolar que sigue hoy vigente. Mi contribución pretende reflexionar sobre la conferencia como práctica social, antes de pasar a una observación de las tareas que comprende la preparación de la misma. El análisis de textos producidos por los alumnos permitirá mostrar algunas de las dificultades a las que se enfrentan, lo que nos llevará a proponer ciertos requisitos que permitan mejorar los resultados de esta actividad.

Datos sobre la autora: Estudios de filología vasca. Trabajos de investigación en el campo del análisis del discurso en el marco del equipo de Didáctica de la lengua de la Universidad de Ginebra. Tesis doctoral sobre el discurso vasco. Actualmente trabaja en un proyecto de investigación sobre los mecanismos de transposición en los manuales de lengua.

Dirección: Universidad de Ginebra, FPSE, 11, Rte de Drize, CH - 1227 Ginebra (Suiza).

(C) PERMISOS PARA CITAR O REPRODUCIR EN OTRAS FUENTES: Se pueden citar libremente hasta 500 palabras. Para reproducir una porción de texto mayor, figuras o ilustraciones, se deberá pedir permiso por escrito a la revista, especificando el uso al que se destina el texto. En todos los casos, se deberá citar el copyright de $C L \& E$. En el caso de artículos o textos que hayan sido a su vez reproducidos en $C L \& E$ los interesados deberán dirigirse tanto a los detentadores del copyright original como a $C L \& E$, en el caso de que se quiera hacer uso de la traducción. FOTOCOPIAS: Para todo lo relacionado con el uso mediante fotocopia del material de esta revista, deberán dirigirse a: CEDRO, C/ José Marañón, 10, 3. Izda. Tel. 5941575 . Fax 4453567 